\title{
Visibility of Type III burst source location as inferred from stereoscopic space observations
}

\author{
M. Y. Boudjada ${ }^{1}$, P. H. M. Galopeau ${ }^{2}$, M. Maksimovic ${ }^{3}$, and H. O. Rucker ${ }^{1}$ \\ ${ }^{1}$ Space Research Institute, Austrian Academy of Sciences, Graz, Austria \\ ${ }^{2}$ Université Versailles St-Quentin, CNRS/INSU, LATMOS-IPSL, Guyancourt, France \\ ${ }^{3}$ LESIA - Observatoire de Paris-Meudon, Meudon, France \\ Correspondence to: M. Y. Boudjada (mohammed.boudjada@oeaw.ac.at)
}

Received: 10 January 2014 - Revised: 4 July 2014 - Accepted: 27 August 2014 - Published: 10 November 2014

\begin{abstract}
We study solar Type III radio bursts simultaneously observed by RPWS/Cassini, URAP/Ulysses and WAVES/Wind experiments. The observations allows us to cover a large frequency bandwidth from $16 \mathrm{MHz}$ down to a few $\mathrm{kHz}$. We consider the onset time of each burst, and estimate the corresponding intensity level. Also we measure the Langmuir frequency as observed on the dynamic spectra recorded by the Ulysses spacecraft. The distances of Wind, Ulysses and Cassini spacecraft, with regard to the Sun, were in the order of $1 \mathrm{AU}, 2.4 \mathrm{AU}$ and $4.5 \mathrm{AU}$, respectively. The spacecraft trajectories were localized in the ecliptic plane in the case of Wind and Cassini, and for Ulysses in the southern hemisphere (i.e. heliocentric latitude higher than $-50^{\circ}$ ). Despite the different locations, the spectral patterns of the selected solar bursts are found to be similar between $10 \mathrm{MHz}$ and $2 \mathrm{MHz}$ but unalike at lower frequency. We discuss the variation of the intensity level as recorded by the three spacecraft. We show that the reception system of each experiment affected the way the Type III burst intensity is measured. Also we attempt to estimate the electron beam along the interplanetary magnetic field where the trajectory is an Archimedean spiral. This leads us to infer on the visibility of the source location with regard to the spacecraft position.
\end{abstract}

\section{Introduction}

Non-thermal radio bursts are caused by energetic electrons from the Sun, which convert part of their energy into electromagnetic radiation via an emission mechanism (Ginzburg and Zheleznyakov, 1958). This mechanism involves the conversion of electron beam excited by Langmuir waves into es- caping radiation at the fundamental $(F)$ and second harmonic $(H)$ of the electron plasma frequency. Direct observation of non-thermal electrons and plasma waves in space, in association with Type III bursts, provided an evidence for the plasma emission mechanism (Lin, 1973). The distribution function of these non-thermal electrons indeed demonstrated the generation of Langmuir waves which derive their energy from the non-thermal electrons; the intensity of the radio bursts depends on the non-thermal electron density and energy (Dulk et al., 1998).

In a dynamic spectrum, the Type III burst appears as an intense band of emission drifting rapidly with $\mathrm{d} f / \mathrm{d} t$ $\sim 100 \mathrm{MHz} \mathrm{s}^{-1}$ from high to low frequencies. Since the corona and the interplanetary (IP) medium are magnetized plasmas, propagation of electrons occurs along open magnetic field lines. Investigations led to a number of advances in studying electron beams as well as inferring the Archimedean spiral structure of the IP magnetic field, (Fainberg and Stone, 1970). Knowledge of the emission directivity is very important when studying the emission mechanism and propagation effects in the medium, particularly at low frequency. At lower frequencies, namely at hectometer and kilometer wavelengths, the widespread visibility of the Type III emission was reported by MacDowall et al. (1982). The visibility is associated to the beaming of the solar radio burst which consists of at least two components: a Gaussian core of half width about $60^{\circ}$, and a very broad halo that extends to $180^{\circ}$ (Dulk et al., 1985). Later the first stereoscopic directivity measurements were reported by Poquerusse et al. (1996) and Hoang et al. (1997), using comparisons of flux densities between the radio receiver on the Ulysses spacecraft and the radio spectrographs ARTEMIS at the highest 


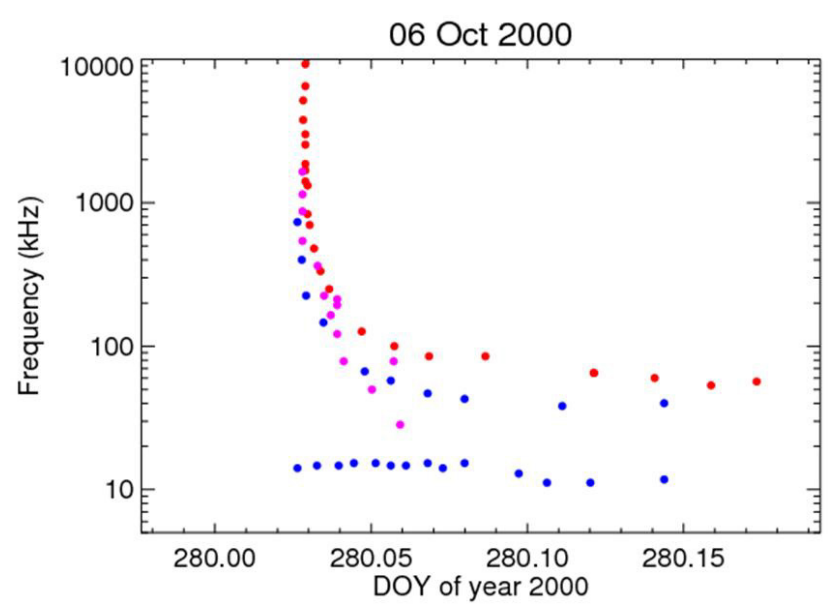

Figure 1. Spectral shape of the Type III solar bursts recorded on 6 October 2000 by Wind/WAVES (red points), Cassini/RPWS (violet points) Ulysses/URAP (blue points) experiments

frequencies, between 100 and $500 \mathrm{MHz}$. More recently, Bonnin et al. (2008) investigated the directivity of solar type III radio bursts at hectometer and kilometer wavelengths using radio data recorded simultaneously by the Wind and Ulysses spacecraft. They derived for the first time the average directivity diagram of Type III bursts in two dimensions, longitude and latitude relative to the emission source. The diagram in longitude was found to shift east of the local magnetic field direction at the source. The angular width and eastward shift of the diagram are observed to increase with decreasing frequencies.

In this paper we attempt to analyze the visibility of solar Type III bursts observed simultaneously by Wind, Ulysses and Cassini spacecraft. In Sect. 2, we describe the radio space experiment and their mutual positions in the ecliptic plane. The method of data investigation is given in Sect. 3 where we consider that the electrons at the origin of the Solar Type III burst follows the interplanetary magnetic field. The main outcomes are discussed in the Sect. 4 .

\section{Radio space experiments}

The data used in this investigation were acquired by URAP, WAVES and RPWS experiments on board Ulysses, Wind and Cassini spacecraft, respectively. The URAP instrument (Stone et al., 1992) measures the wave electric fields in two bands. The high-band receivers between 52 and $940 \mathrm{kHz}$, and the low-band receivers cover the range from 1.25 to $48.50 \mathrm{kHz}$. On the Wind satellite we investigate the data recorded by the super heterodyne receivers RAD1 and RAD2, respectively, in the frequency range $20-1024 \mathrm{kHz}$ and 1.075-13.825 MHz (Bougeret et al. , 1995). The RPWS experiment is designed to measure the electric and magnetic fields of radio emissions and plasma waves across a broad

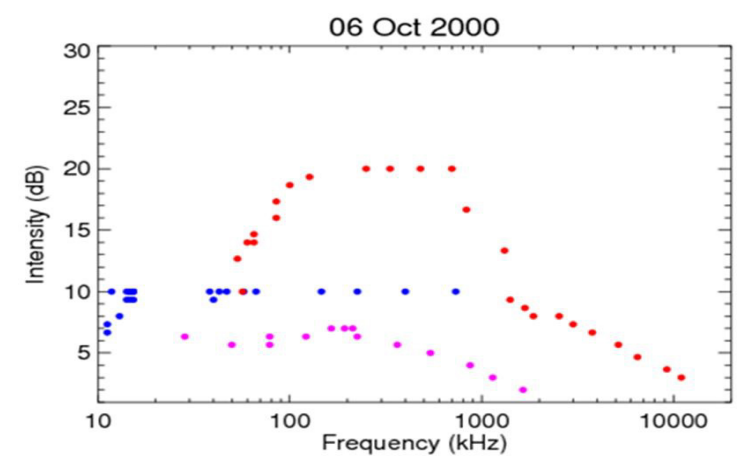

Figure 2. Variation of the intensity level (expressed in $\mathrm{dB}$ ) versus the frequency range (in $\mathrm{kHz}$ ) for the solar type III burst recorded on 6 October 2000

range of frequencies (Gurnett et al. , 2004). The high frequency receiver (HFR) enables the analysis of incoming radio waves by combining signals from three $10 \mathrm{~m}$ long antenna elements. The covered frequency bandwidth is from 3.5 to $16 \mathrm{MHz}$.

In this study, we combine the simultaneous observations of solar Type III radio bursts observed by the three spacecraft. We consider solar Type III bursts observed from September 2000 to March 2001. During this period, Wind and Cassini were nearly orbiting in the ecliptic plane, and Ulysses was in the Southern hemisphere at heliocentric latitude higher than $-50^{\circ}$. The distances of Wind, Ulysses and Cassini spacecrafts, with regard to the Sun, were in the order of $1 \mathrm{AU}$, 2.4 AU and 4.5 AU, respectively (see Fig. 3).

\section{Method of data investigation}

We study more than 100 solar bursts simultaneously observed by the three spacecraft, from September 2000 to March 2001. The method of data investigations consists of the analysis of: (a) the variation of the observed frequency versus the time, (b) the intensity level versus the frequency, and (c) the Archimedean spiral at the origin of the solar burst. Hereafter we use the solar Type III burst observed on 6 October 2000 as an example for the data investigation.

In Fig. 1 we show the Type III burst observed by the three spacecraft on 6 October 2000. The blue, red and violet colors are associated, respectively, to Ulysses, Wind and Cassini observations. Despite the different spacecraft locations with regards to the Sun, the spectral shapes are found to be similar in the frequency range between 13 and $2 \mathrm{MHz}$, and look different at lower frequencies, less than $1 \mathrm{MHz}$. The frequency at about $15 \mathrm{kHz}$ is the Langmuir frequency observed by Ulysses spacecraft. It is the lowest observed frequency. It is produced by thermal motions of the electrons in the vicinity of the spacecraft (Meyer-Vernet and Perche, 1989).

Figure 2 shows the variation of the intensity level (in $\mathrm{dB}$ ) vs. the frequency (in $\mathrm{kHz}$ ). The Wind observations (in red 
colors in Fig. 2) showed an increase of the intensity level emission from 10 to $20 \mathrm{~dB}$ between 60 and $100 \mathrm{kHz}$. Followed by a maximum of intensity of about $20 \mathrm{~dB}$ in the frequency range $100 \mathrm{kHz}$ to $1 \mathrm{MHz}$. Then a clear decrease of the intensity level from 20 to $3 \mathrm{~dB}$ at high frequency (i.e. between 1 and $13 \mathrm{MHz}$ ). The Ulysses observations (blue color in Fig. 2) showed an increase from 5 to $10 \mathrm{~dB}$ at very low frequency (10 to $15 \mathrm{kHz}$ ). This is followed by a maximum about $10 \mathrm{~dB}$ in the frequency range from $15 \mathrm{kHz}$ to about $900 \mathrm{kHz}$. The Cassini intensity level (in violet color in Fig. 2) has a maximum of about $6 \mathrm{~dB}$ between 30 and $200 \mathrm{kHz}$, followed by a decrease to about $2 \mathrm{~dB}$ in the frequency range $200 \mathrm{kHz}$ to $2 \mathrm{MHz}$. We note that the intensity level decreases when the distance to the Sun increases. This explains the maximum of $20 \mathrm{~dB}$ observed by Wind, and the lowest level of $6 \mathrm{~dB}$ for Cassini. Instrumental effect seems to be at the origin of the increase of the Wind intensity level between 60 and $100 \mathrm{kHz}$. The two other spacecraft show a constant intensity level in the bandwidth $60-100 \mathrm{kHz}$. Also the decrease of the intensity above $200 \mathrm{kHz}$ in the Cassini observations is due to the reception system. Wind and Ulysses show a constant level, and no decrease between 200 and $900 \mathrm{kHz}$. Furthermore the decrease of Wind intensity above $1 \mathrm{MHz}$ can be explained by an instrumental effect because the RAD2 and RAD1 frequency bandwidth started and ended at about $1 \mathrm{MHz}$.

Further we analyze the geometry of the solar burst source with regards to the spacecraft locations. We first use the empirical formula which allows us to estimate the distance at which occur the observed frequency. We consider the empirical formula derived by Bougeret et al. (1984) using the Helios 1 and 2 in situ measurements. For this we use:

$f_{\mathrm{p}}=22.5 r^{-1.05}$

where $f_{\mathrm{p}}$ is the local plasma frequency and $r$ is the heliocentric distance expressed in AU. Then we consider that the electrons at the origin of the Solar Type III bursts follow the interplanetary magnetic field as shown in Fig. 3. The horizontal and vertical axes are expressed in Solar radii. The trajectory is an Archimedean spiral contained in the ecliptic plane. The equation of the curves in Fig. 3 is given by:

$\phi=\frac{\Omega}{V_{\mathrm{SW}}} \frac{r}{215}$

where, $\Omega, V_{\mathrm{SW}}$, and $r$ are, respectively, the solar angular rotation (in $\mathrm{rad} \mathrm{s}^{-1}$ ), solar wind speed (in $\mathrm{km} \mathrm{s}^{-1}$ ) and the distance to the Sun in solar radii (in $\mathrm{km}$ ). This equation includes the foot of the spiral on the Sun's surface, and gives the orientation of the spiral with respect to the Sun-Earth line. We have used a list of sunspot observations to find the location of the active regions on the Sun. This list is provided by the National Geophysical Data Center (NGDC) by FTP area (ftp.ngdc.noaa.gov). We have found two active regions on the Sun for the considered event of 6 October 2000, at about 00:50 UT, one in eastern part and other in the western one. Since this active region is simultaneously observed by

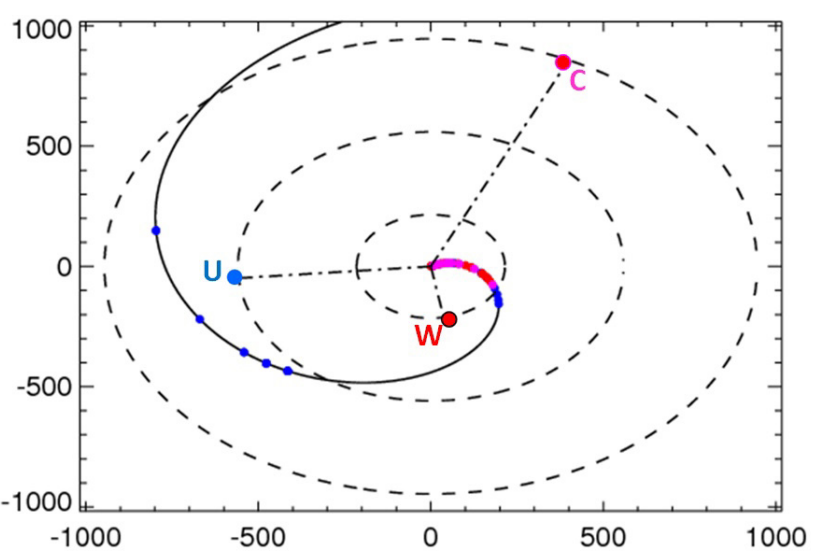

Figure 3. Archimedean spiral associated to the electrons at the origin of the solar type III bursts showed in Fig. 1. The higher frequencies $(\sim 10 \mathrm{MHz})$ are supposed to be generated in the solar corona and the lower one $(\sim 100 \mathrm{kHz})$ in the interplanetary medium. The dashed and dash-dotted lines indicated, respectively, the spacecraft orbits and the positions of Wind $(W)$, Ulysses $(U)$ and Cassini $(C)$ on 6 October 2000.

Wind and Cassini spacecraft (both on the western part of the Sun), we have decided to selec the source region located at $45^{\circ} \mathrm{W}$. In Eq. (2), we assume a constant solar wind speed of $400 \mathrm{~km} \mathrm{~s}^{-1}$.

The spacecraft orbits are shown in Fig. 3 where their positions on 6 October 2000 are indicated with the letters " $W$ " for Wind, " $U$ " for Ulysses and " $C$ " for Cassini. It is clear that the active region observed in the western part of the Sun is the real source location of the observed Type III burst. We can assume that the emission diagram is a cone with a symmetrical axis along the magnetic field line emerging from the Sun at about $45^{\circ} \mathrm{W}$. The cone opening angle can be estimated as about $90^{\circ}$. At lower frequencies (less than $1 \mathrm{MHz}$ ) it is not evident to presume the shape of the emission diagram. The lower spectrum of the Type III burst was observed by the three spacecraft which means a large opening angle, more than $180^{\circ}$. However we note that the shapes are different, as recorded by each spacecraft.

\section{Results and preliminary conclusion}

We investigate more than 100 solar bursts observed from September 2000 to March 2001. We find that $44 \%$ of the solar bursts are associated to active regions localized in the western part of the Sun which is visible to the Earth. A small part of about $15 \%$ is linked to active regions in the eastern part of the Sun. And the rest of the bursts $(41 \%)$ can not be associated to active regions on the Sun. This means that the corresponding active regions, can't be observed from the Earth, and should be behind the Sun and on the western limb of our star. Only in such configurations Wind and Cassini can detect simultaneously the radio emission of the solar burst. 
Also we find that the spectral shapes (as shown in Fig. 1) of the Solar bursts are similar in the frequency range between 13 and $2 \mathrm{MHz}$. This leads us to presume that the radio wave is not disturbed by the plasma environment in the solar corona and the interplanetary medium. This is not the case at frequencies lower than $1 \mathrm{MHz}$ where we note different spectral shapes (as shown in Fig. 1). This is clear evidence of the presence of electron density inhomogeneity which alters the radio wave propagation in the interplanetary medium. Our result is in agreement with previous investigations (Dulk et al., 1985; Lecacheux et al, 1989). The origin of the different spectral shapes may be due to a high degree of scattering of radio waves originating at or near the local plasma frequency.

Further investigations should take into considerations other models of electron density (e.g. Mann et al., 1999). Also more bursts will be analyzed with the aim to combine our results to the works of Bonnin et al. (2008) and Krupar et al (2012). The future European mission Solar Orbiter (Boudjada et al., 2005) will give us the possibility to investigate the electron density at very close distances to the Sun during the nominal mission phase (duration of about 2.88 years), from about 0.2 to $0.9 \mathrm{AU}$ (Marsch et al., 2001).

Acknowledgements. The authors are grateful to J. L. Bougeret (PI of the Wind experiment), D. A. Gurnett (PI of the RPWS experiment), and R. J. MacDowall (PI of the URAP experiment) for making the data available for this work.

Edited by: G. Mann

Reviewed by: two anonymous referees

\section{References}

Bonnin, X., Hoang, S., and Maksimovic, M: The directivity of solar type III bursts at hectometer and kilometer wavelengths: WindUlysses observations, Astron. Astrophys., 489, 419-427, 2008.

Boudjada, M. Y., Macher, W., Rucker, H. O., and Fischer, G.: Solar Orbiter: Physical aspects towards a better knowledge of the solar corona, Adv. Space Res., 36, 1439, doi:10.1016/j.asr.2005.05.118, 2005.

Bougeret, J.-L., Kaiser, M. L., Kellogg, P. J., Manning, R., Goetz, K., Monson, S. J., Monge, N., Friel, L., Meetre, C. A., Perche, C., Sitruk, L., and Hoang, S.: WAVES: The radio and plasma wave investigation on theWind spacecraft, Space Sci. Rev., 71, 231-263, 1995.

Bougeret, J. L., King, J. H., and Schwenn, R.: Solar radio burst and in situ determination of interplanetary electron density, Sol. Phys., 90, 401-412, 1984.

Dulk, G. A., Leblanc, Y., Robinson, P. A., Bougeret, J. L., and Lin, R. P.: Electron beams and radio waves of solar Type III bursts, J. Geophys. Res., 103, 17223-17233, 1998.

Dulk, G. A., Steinberg, J. L., Lecacheux, A., Hoang, S., and MacDowall, R. J.: The visibility of type III radio bursts originating behind the sun, Astron. Astrophys., 150, L28-L30, 1985.
Fainberg, J. and Stone, R. G.: Type III solar radio burst storms observed at low frequencies, I. Storm morphology, Sol. Phys., 15, 222-233, 1970.

Ginzburg, V. L. and Zheleznyakov, V. V: On the Possible Mechanisms of Sporadic Solar Radio Emission - Radiation in an Isotropic Plasma, Astr. Zh., 35, 653-668, 1958.

Gurnett, D. A., Kurth, W. S., Kirchner, D. L., Hospodarsky, G. B., Averkamp, T. F., Zarka, P., Lecacheux, A., Manning, R., Roux, A., Canu, P., Cornilleau-Wehrlin, N., Galopeau, P., Meyer, A., Bostrom, R., Guastafsson, G., Wahlund, J.-E., Aahlen, L., Rucker, H. O., Ladreiter, H. P., Macher, W., Woolliscroft, L. J. C., Alleyne, H., Kaiser, M. L., Desch, M. D., Farrell, W. M., Harvey, C. C., Louarn, P., Kellogg, P. J., Goetz, K., and Pedersen, A.: The Cassini radio and plasma wave science investigation, Space Sci. Rev., 114, 395-463, 2004.

Hoang, S., Poquerusse, M., and Bougeret, J. L.: The directivity of solar kilometric Type III bursts: Ulysses-Artemis observations in and out of the ecliptic plane, Sol. Phys., 172, 307-316, 1997.

Krupar, V., Santolik, O., Cecconi, B., Maksimovic, M., Bonnin, X., Panchenko, M., and Zaslavsky, A.: Goniopolarimetric inversion using SVD: An application to type III radio bursts observed by STEREO, J. Geophys. Res., 117, A06101, doi:10.1029/2011JA017333, 2012.

Lecacheux, A., Steinberg, J. L., Hoang, S., and Dulk, G. A.: Characteristics of type III bursts in the solar wind from simultaneous observations on board ISEE-3 and Voyager, Astron. Astrophys., 217, 237-250, 1989.

Lin, R. P., Evans, L. G., and Fainberg, J.: Simultaneous observations of fast solar electrons and Type III radio burst emission near 1 AU, Astrophys. J., 14, 191-198, 1973.

MacDowall, R. J., Stone, R. G., and Kaiser, M. L.: Kilometric Type III burst directivity from simultaneous spacecraft observations, Bulletin of the American Astronomical Society, 14, 607-607, 1982.

Mann, G., Jansen, F., MacDowall, R. G., Kaiser, M. L., and Stone, R. G.: A heliospheric density model and type III radio bursts, Astron. Astrophys., 348, 614-620, 1999.

Marsch, E., Harrison, R., Pace, O. Antonucci, E., Bochsler, P., Bougeret, J.-L., Fleck, B., Langevin, Y., Marsden, R., Schwenn, R., and Vial, J.-C.: Solar Orbiter, a high resolution mission to the Sun and inner heliosphere, Physical aspects towards a better knowledge of the solar corona, In: Solar encounter. Proceedings of the First Solar Orbiter Workshop, edited by: Battrick, B. and Sawaya-Lacoste, H., ESA SP-493, Noordwijk: ESA Publications Division, ISBN 92-9092-803-4, 2001.

Meyer-Vernet, N. and Perche, C.: Tool kit for antennas in plasmas, J. Geophys. Res., 94, 2405-2415, 1989.

Poquérusse, M., Hoang, S., Bougeret, J. L., and Moncuquet, M.: Ulysses- ARTEMIS radio observation of energetic flare electrons, in Amer. Inst. Phys. Conf. Ser. 382, edited by: Winterhalter, D., Gosling, J. T., Habbal, S. R., Kurth, W. S., and Neugebauer, M., 62-65, 1996.

Stone, R. G., Pedersen, B. M., Harvey, C. C., Canu, P., CornilleauWehrlin, N., Desch, M. D., de Villedary, C., Fainberg, J., Farrell, W. M., and Goetz, K.: ULYSSES radio and plasma wave observations in the Jupiter environment, Science, 257, 1524-1531, 1992. 